\title{
Estatinas e COVID-19: Suspender ou não Suspender? Eis a Questão!
}

\section{Statins and COVID-19: To Suspend or Not to Suspend? That is the Question!}

\author{
Filipe Ferrari ${ }^{10}$ e Raul D. Santos ${ }^{2,3}$ \\ Programa de Pós-Graduação em Cardiologia e Ciências Cardiovasculares, Universidade Federal do Rio Grande do Sul, Hospital de Clínicas de \\ Porto Alegre, ${ }^{1}$ Porto Alegre, $R S$ - Brasil \\ Unidade Clínica de Lípides, Instituto do Coração (InCor), Hospital da Faculdade de Medicina da Universidade de São Paulo, ${ }^{2}$ São Paulo, SP - Brasil \\ Hospital Israelita Albert Einstein, ${ }^{3}$ São Paulo, SP - Brasil
}

\section{Introdução}

Em meio a tantas incertezas que permeiam a nova doença de coronavírus 2019 (COVID-19), as evidências relacionando a presença de dislipidemia à gravidade da doença e às consequentes implicações prognósticas ainda são escassas. Em maio de 2020, um estudo chinês retrospectivo investigou a associação entre as mudanças nos níveis de colesterol e o prognóstico em aproximadamente 600 pacientes com COVID-19, pareados por idade e sexo com controles saudáveis. Primeiramente, observou-se que os níveis de colesterol de lipoproteína de baixa densidade (LDL-C) e colesterol total foram significativamente mais baixos em pacientes com COVID-19. Em segundo lugar, houve uma tendência para os níveis de LDL-C e de colesterol total diminuirem à medida que a gravidade da infecção aumentava (leve, grave e crítica, respectivamente). ${ }^{1}$ No mesmo estudo, os níveis de colesterol de lipoproteína de alta densidade (HDL-C) também diminuíram em casos graves. Foram observados dados parecidos por Fan et al., ${ }^{2}$ onde os níveis de LDL-C foram inversamente associados à gravidade da COVID-19. Esses dados sugeriram uma possível relação entre níveis baixos de colesterol e piora da infecção por COVID-19. Além disso, estudos experimentais têm mostrado que as estatinas podem aumentar a abundância da enzima conversora de angiotensina 2 (ECA2), o que pode contribuir parcialmente para a entrada do vírus na célula e o aumento do risco de infectividade. ${ }^{3}$

Com base nesses achados prévios, foi hipotetizado que o uso de terapias hipolipemiantes como as estatinas poderiam agravar a infecção por COVID-19. No entanto, sabe-se que os níveis de colesterol sérico podem cair em pacientes com infecções virais ou bacterianas ativas, ${ }^{4,5}$ uma vez que o LDL e o HDL desempenham um papel no sistema imunológico. ${ }^{6}$ Por outro lado, a hiperlipidemia pode comprometer a resposta imunológica e agravar ainda mais o estado inflamatório dos pacientes com COVID-19, aumentando o risco

\section{Palavras-chave}

COVID-19; Coronavírus; Betacoronavírus; Pandemia; Colesterol; Dislipidemias; Infecção; Inibidores de Hidroximetilglutaril-CoA Redutases; Lipoproteínas.

Correspondência: Raul D. Santos •

Av. Albert Einstein, 627. CEP 05652-900, São Paulo, SP - Brasil

E-mail: rauldsf@gmail.com

Artigo recebido em 10/08/2020, revisado em 28/09/2020, aceito em 10/10/2020

DOI: https://doi.org/10.36660/abc.20200949 cardiovascular. ${ }^{7}$ Desta maneira, surge a seguinte pergunta: as estatinas deveriam ou não ser suspensas em pacientes com COVID-19?

COVID-19, Infecções, Trombose e Estatinas

\section{Evidências de Benefícios Potenciais}

Além de diminuir as lipoproteínas pró-aterogênicas, as estatinas apresentam outros efeitos sistêmicos bem documentados, como a melhora da disfunção endotelial e as propriedades anti-inflamatórias e anti-trombóticas que levam à estabilização das placas ateroscleróticas. ${ }^{8}$ Metaanálises de ensaios clínicos randomizados mostraram que as estatinas podem reduzir significativamente as concentrações de proteína $\mathrm{C}$ reativa, ${ }^{9}$ antígeno do fator de von Willebrand ${ }^{10}$ e endotelina-1. ${ }^{11}$

Um estudo observacional com 3.043 pacientes hospitalizados pelo vírus influenza encontrou menor risco de mortalidade naqueles que estavam em uso de estatinas, antes ou durante a hospitalização (odds ratio [OR] ajustado 0,59). ${ }^{12}$ Também foi observado um benefício das estatinas em pacientes hospitalizados com pneumonia viral, resultando em menor mortalidade e necessidade de intubação (OR 0,26). ${ }^{13}$

Dado o estado pró-inflamatório e pró-trombótico observado em pacientes com COVID-19 mais grave, as características dessas drogas podem ser importantes para esses pacientes.

A Tabela 1 mostra detalhes de alguns estudos que examinaram os efeitos das estatinas em pacientes com infecções virais e COVID-19.

Em um estudo de coorte retrospectivo da Bélgica, De Spiegeleer et al. ${ }^{14}$ avaliaram 154 pessoas idosas (idade média: 86 anos) que contraíram COVID-19, observando uma tendência significativa para ausência de sintomas naqueles que recebiam estatinas anteriormente (OR 2,91; intervalo de confiança (IC) 95\%, 1,27 a 6,71). Isto permaneceu estatisticamente significativo mesmo após o ajuste para covariáveis (OR 2,65; IC 95\%, 1,13 a 6,68).

Outro estudo retrospectivo de aproximadamente 14.000 pacientes com COVID-19 encontrou menor risco de mortalidade com o uso prévio de estatinas. Neste estudo, 1.219 pacientes estavam recebendo estatinas e a mortalidade geral em 28 dias neste grupo foi de 5,2\%, enquanto no grupo sem estatinas foi de $9,4 \%$ (hazard ratio [HR] ajustado 0,58; IC 95\%, 0,43 a 0,80; $p=0,001) \cdot{ }^{15}$ Em outro estudo com 87 pacientes com COVID-19 internados na unidade de 


\section{Carta Científica}

\section{Tabela 1 - Evidências de possíveis benefícios das estatinas no cenário de doenças virais, bem como na COVID-19}

\begin{tabular}{|c|c|c|c|c|c|}
\hline Estudo & $\begin{array}{l}\text { Desenho do } \\
\text { Estudo }\end{array}$ & Pacientes e Doença & $\begin{array}{c}\text { Total (N) } \\
\text { Idade média }\end{array}$ & Ajuste para covariáveis & Resultados \\
\hline \multirow[t]{2}{*}{$\begin{array}{l}\text { Vandermeer } \\
\text { et al. } 2011^{12}\end{array}$} & \multirow[t]{2}{*}{ Multiestado } & \multirow[t]{2}{*}{$\begin{array}{l}\text { Pacientes hospitalizados } \\
\text { com infecções pelo vírus } \\
\text { influenza }\end{array}$} & \multirow[t]{2}{*}{$\begin{array}{l}3.043 \\
70 \text { anos }\end{array}$} & \multirow[t]{2}{*}{$\begin{array}{c}\text { Idade, raça, DCV, doença } \\
\text { pulmonar e renal, vacinação contra } \\
\text { influenza e administração de } \\
\text { medicamentos antivirais }\end{array}$} & $\begin{array}{l}\text { Estatinas antes ou durante a } \\
\text { hospitalização versus nenhuma estatina } \\
\text { foram associadas a chances de } \\
\text { proteção contra a mortalidade } \\
\text { em } 30 \text { dias }\end{array}$ \\
\hline & & & & & OR ajustado 0,59; IC 95\%, 0,30 a 0,92 \\
\hline \multirow[t]{2}{*}{$\begin{array}{l}\text { Henry } \\
\text { et al. } 2018^{13}\end{array}$} & \multirow[t]{2}{*}{ Retrospectivo } & \multirow[t]{2}{*}{$\begin{array}{c}\text { Pacientes com pneumonia } \\
\text { viral }\end{array}$} & \multirow[t]{2}{*}{$\begin{array}{c}539 \\
64 \text { anos }\end{array}$} & \multirow[t]{2}{*}{ NA } & $\begin{array}{l}\text { Estatinas continuadas no hospital } \\
\text { versus descontinuação reduziram } \\
\text { mortes e/ou necessidade de intubação } \\
\text { durante a internação hospitalar }\end{array}$ \\
\hline & & & & & OR 0,$26 ;$ IC $95 \%, 0,08$ a 0,$81 ; p=0,02$ \\
\hline \multirow[t]{2}{*}{$\begin{array}{l}\text { De } \\
\text { Spiegeleer } \\
\text { et al. } 2020^{14}\end{array}$} & \multirow[t]{2}{*}{$\begin{array}{l}\text { Coorte multicêntrica } \\
\text { retrospectiva }\end{array}$} & \multirow[t]{2}{*}{$\begin{array}{l}\text { Sujeitos positivos para } \\
\text { COVID-19 }\end{array}$} & \multirow[t]{2}{*}{$\begin{array}{c}154 \\
86 \text { anos }\end{array}$} & \multirow[t]{2}{*}{$\begin{array}{l}\text { Idade, sexo, estado funcional, } \\
\text { hipertensão e diabetes mellitus }\end{array}$} & $\begin{array}{l}\text { O uso de estatinas foi relacionado } \\
\text { à ausência de sintomas durante } \\
\text { COVID-19 }\end{array}$ \\
\hline & & & & & $\begin{array}{l}\text { OR ajustado } 2,65 ; \text { IC } 95 \% \\
1,13 \text { a } 6,68 ; p=0,028\end{array}$ \\
\hline \multirow[t]{2}{*}{$\begin{array}{l}\text { Zhang } \\
\text { et al. } 2020^{15}\end{array}$} & \multirow[t]{2}{*}{ Retrospectivo } & \multirow[t]{2}{*}{$\begin{array}{l}\text { Pacientes hospitalizados } \\
\text { por COVID-19 }\end{array}$} & \multirow[t]{2}{*}{$\begin{array}{c}13.981 \\
58 \text { anos }\end{array}$} & \multirow[t]{2}{*}{$\begin{array}{l}\text { Idade, sexo e SpO2 no } \\
\text { momento da admissão }\end{array}$} & $\begin{array}{l}\text { Uso de estatinas versus nenhuma } \\
\text { estatina foi correlacionado à redução } \\
\text { no risco de mortalidade por todas as } \\
\text { causas em } 28 \text { dias }\end{array}$ \\
\hline & & & & & $\begin{array}{l}\text { HR ajustado } 0,58 ; \text { IC } 95 \%, 0,43 \text { a } \\
\qquad 0,80 ; p=0,001\end{array}$ \\
\hline
\end{tabular}

Idade, hipertensão, DCV, ventilação mecânica invasiva, frequência respiratória $>30$, $\mathrm{SpO} 2<94 \%, \mathrm{PaO} 2 / \mathrm{FiO} 2<300$

Rodriguez-

Nava et al.

$2020^{16}$

$\begin{array}{cc}\text { Coorte } & \text { Pacientes com COVID-19 } \\ \text { retrospectiva } & \text { admitidos à unidade de } \\ \text { terapia intensiva }\end{array}$

87

68 anos
$\mathrm{mmHg}$ ou infiltrados pulmonares $>$ $50 \%$, número de comorbidades e

outras terapias adjuvantes (incluindo hidroxicloroquina, esteróides intravenosos, azitromicina, tocilizumabe colchicina e antibióticos)
O uso de estatina (especificamente atorvastatina) reduziu a progressão à morte

HR ajustado 0,38, IC 95\%, 0,18 a 0,77; $p=0,008$

\begin{tabular}{|c|c|c|c|c|}
\hline $\begin{array}{l}\text { Daniels et al. } \\
2020^{17}\end{array}$ & $\begin{array}{l}\text { Unicêntrico } \\
\text { retrospectivo }\end{array}$ & $\begin{array}{l}\text { Pacientes hospitalizados } \\
\text { por COVID-19 }\end{array}$ & $\begin{array}{c}170 \\
59 \text { anos }\end{array}$ & $\begin{array}{l}\text { Idade, sexo, obesidade, hipertensão, } \\
\text { diabetes, doença renal crônica e DCV }\end{array}$ \\
\hline
\end{tabular}

Uso de estatinas antes da admissão reduziu o desenvolvimento de doença grave

OR ajustado 0,29; IC 95\%, 0,11 a 0,$71 ; p=0,009$

O uso de estatinas aumentou a taxa de recuperação da COVID-19 entre os indivíduos que ainda não haviam experimentado doença grave

HR ajustado por causa específica para recuperação 2,69; IC 95\%, 1,36 a 5,33; $p=0,004$

O uso de estatinas diminuiu o risco de ventilação mecânica invasiva

OR ajustado 0,45; IC 95\%, 0,20 a 0,$99 ; p=0,048$

OR: odds ratio; HR: hazard ratio; IC: intervalo de confiança; $\mathrm{SpO}_{2}$ : saturação periférica de oxigênio: DCV: doença cardiovascular; NA: não aplicável. 
terapia intensiva, uma progressão mais lenta para a morte foi encontrada naqueles que receberam atorvastatina. ${ }^{16}$

Daniels et al., ${ }^{17}$ por meio de um estudo retrospectivo de centro único, encontraram um risco reduzido de COVID-19 grave em pacientes que estavam recebendo estatinas antes da admissão (OR ajustado 0,29) e um tempo mais rápido de recuperação entre aqueles sem doença grave (HR ajustado para recuperação 2,69). Além disso, em um estudo de coorte retrospectivo de pacientes hospitalizados com COVID-19 ( $\mathrm{N}=249$ ) nos Estados Unidos, o uso de estatinas foi correlacionado à diminuição do risco de ventilação mecânica invasiva (OR ajustado 0,45). ${ }^{18}$

Certamente, os estudos citados são severamente limitados por seu desenho retrospectivo; esses dados, apesar de favoráveis ao uso de estatinas em infecções virais, são apenas geradores de hipóteses, podendo estar sujeitos a um viés de seleção de indivíduos que recebem melhores cuidados. A questão que se segue é: há alguma evidência de que as estatinas possam prevenir doenças infecciosas? Em uma análise post hoc de pacientes incluídos no ensaio JUPITER, ${ }^{19}$ que randomizou 17.802 indivíduos com LDL-C < $130 \mathrm{mg} /$ dL e proteína $C$ reativa de alta sensibilidade $\geq 2,0 \mathrm{mg} / \mathrm{L}$ para receber rosuvastatina $20 \mathrm{mg} / \mathrm{dia}$ ou placebo, seguidos por tempo mediano de 1,9 anos, Novack et al. ${ }^{20}$ observaram que o uso de estatinas reduziu, embora modestamente, a incidência de pneumonia (HR 0,83, IC 95\%, 0,69 a 1,00). Esses resultados, que merecem ser comprovados em um ensaio adequadamente delineado, sugerem que as estatinas possam reduzir o risco de pneumonia devido a possíveis efeitos benéficos leves anti-inflamatórios, antioxidantes, imunomoduladores, anti-apoptóticos e endoteliais, segundo os autores. ${ }^{18} \mathrm{E}$ incerto se isso beneficiaria pacientes com COVID-19.

Além das complicações pulmonares, o SARS-CoV-2 também pode induzir a trombose. ${ }^{21}$ As estatinas teriam efeitos benéficos nestes casos? Em uma análise pré-especificada do mesmo ensaio JUPITER, ${ }^{19}$ foi analisado o impacto da rosuvastatina na primeira ocorrência de embolia pulmonar ou de tromboembolismo venoso. Embora não tenha havido diferenças nas taxas de embolia pulmonar entre os grupos (rosuvastatina e placebo), o grupo que recebeu a estatina apresentou uma redução de $43 \%$ nas taxas de tromboembolismo venoso (HR 0,57; IC 95\%, 0,37 a 0,86; $\mathrm{p}=0,007) \cdot{ }^{22}$ Além disso, uma metanálise de 13 estudos de coorte observacionais $(N=3.148 .259)$ e 23 ensaios clínicos randomizados ( $N=118.464)$ mostrou que, tanto em estudos de coorte observacionais quanto em ensaios clínicos randomizados, houve uma redução no risco de trombose venosa profunda, mas não de embolia pulmonar, quando o uso de estatina foi comparado com os controles (risco relativo [RR] 0,75; IC 95\%, 0,65 a 0,87; p < 0,0001; 0,85; IC 95\%, $0,73$ a 0,$99 ; p=0,038)$. Um benefício maior também foi encontrado para o risco de tromboembolismo venoso com o uso de rosuvastatina em comparação com outras estatinas (RR 0,57; IC 95\%, 0,22 a 0,75; $p=0,015) .{ }^{23}$ Possíveis mecanismos para explicar esses resultados incluem os efeitos das estatinas sobre fatores pró-trombóticos, como a redução do dímero D, fator VIII, ${ }^{24}$ inibidor do ativador do plasminogênio tipo 1 e níveis de fator tecidual, bem como a diminuição da agregação plaquetária e o aumento da expressão de trombomodulina. ${ }^{25}$ A Figura 1 apresenta alguns mecanismos propostos onde as estatinas podem atuar como agentes anti-trombóticos e antiinflamatórios e podem exercer efeitos favoráveis em pacientes com COVID-19.

Visto que uma porção não negligenciável de pacientes infectados pelo SARS-CoV-2 (especialmente os pacientes mais graves) pode apresentar alterações no sistema de coagulação e uma alta taxa de tromboembolismo venoso, ${ }^{26}$ a manutenção das estatinas pode melhorar o prognóstico desses indivíduos. No entanto, da mesma forma que as possíveis propriedades anti-infecciosas, isso também precisa ser confirmado em ensaios clínicos randomizados.

\section{Suspensão de Estatinas e Aumento do Risco de Eventos Cardiovasculares?}

A preocupação de que os níveis baixos de colesterol possam ser deletérios para pacientes com COVID-19 pode levar à suspensão inadequada de medicamentos hipolipemiantes em pacientes com alto risco de doença cardiovascular. As estatinas são a base fundamental para a terapia hipolipemiante com o objetivo de reduzir o risco de doença arterial coronariana (DAC); como grupo, as estatinas constituem um dos medicamentos mais prescritos no mundo. A metanálise do Cholesterol Treatment Trialists (CTT ${ }^{27}$ mostrou que para cada redução de 1,0 mmol/L ( $40 \mathrm{mg} / \mathrm{dL})$ de LDL-C, houve uma redução de $10 \%$ na mortalidade por todas as causas (RR 0,90, IC 95\%, 0,87 a 0,93; p < 0,0001), além de uma redução de $20 \%$ nas mortes por DAC (RR 0,80; IC 99\%, $0,74$ a 0,$87 ; p<0,0001)$.

Um cenário importante em que a suspensão de estatinas pode ser deletéria é durante o período inicial após um evento de síndrome coronariana aguda. Nesse cenário, a adição e manutenção de estatinas são fundamentais, e a suspensão do medicamento pode aumentar os riscos dos pacientes. Nesse sentido, um estudo observacional brasileiro com 249 pacientes observou um efeito inflamatório rebote na fase aguda do infarto do miocárdio (IM) após a retirada das estatinas. Sposito et al. ${ }^{28}$ verificaram que, no começo do estudo, os pacientes que recebiam estatinas apresentavam valores de proteína C-reativa mais baixos quando comparados aos que não recebiam, antes do início do IM. No quinto dia após o IM, a mediana da proteína $C$ reativa foi significativamente mais alta no grupo onde as estatinas foram suspensas..$^{28} \mathrm{Em}$ adição a isso, em uma análise de pacientes que apresentaram com DAC e dor no peito durante as últimas 24 horas no estudo PRISM $^{29}$ (N $=1.616$ ), Heeschen et al. ${ }^{30}$ relataram que o uso de estatinas reduziu a taxa de eventos após 30 dias, em comparação com os pacientes sem esses medicamentos (HR ajustado 0,49, IC 95\%, 0,21 a 0,86). Quando as estatinas foram suspensas após a admissão, o risco cardíaco aumentou (OR 2,93; IC $95 \%, 1,64$ a 6,27; $p=0,005)$ e, embora não tenha sido estatisticamente significativo, houve uma tendência de maior risco em comparação com os pacientes que nunca receberam estatinas (OR 1,69; IC 95\%, 0,92 a 3,56). ${ }^{29}$ Portanto, a retirada desses medicamentos deve ser vista com extrema cautela, principalmente após um evento coronariano agudo, pois pode levar ao surgimento de complicações, piorando o prognóstico dos pacientes. 


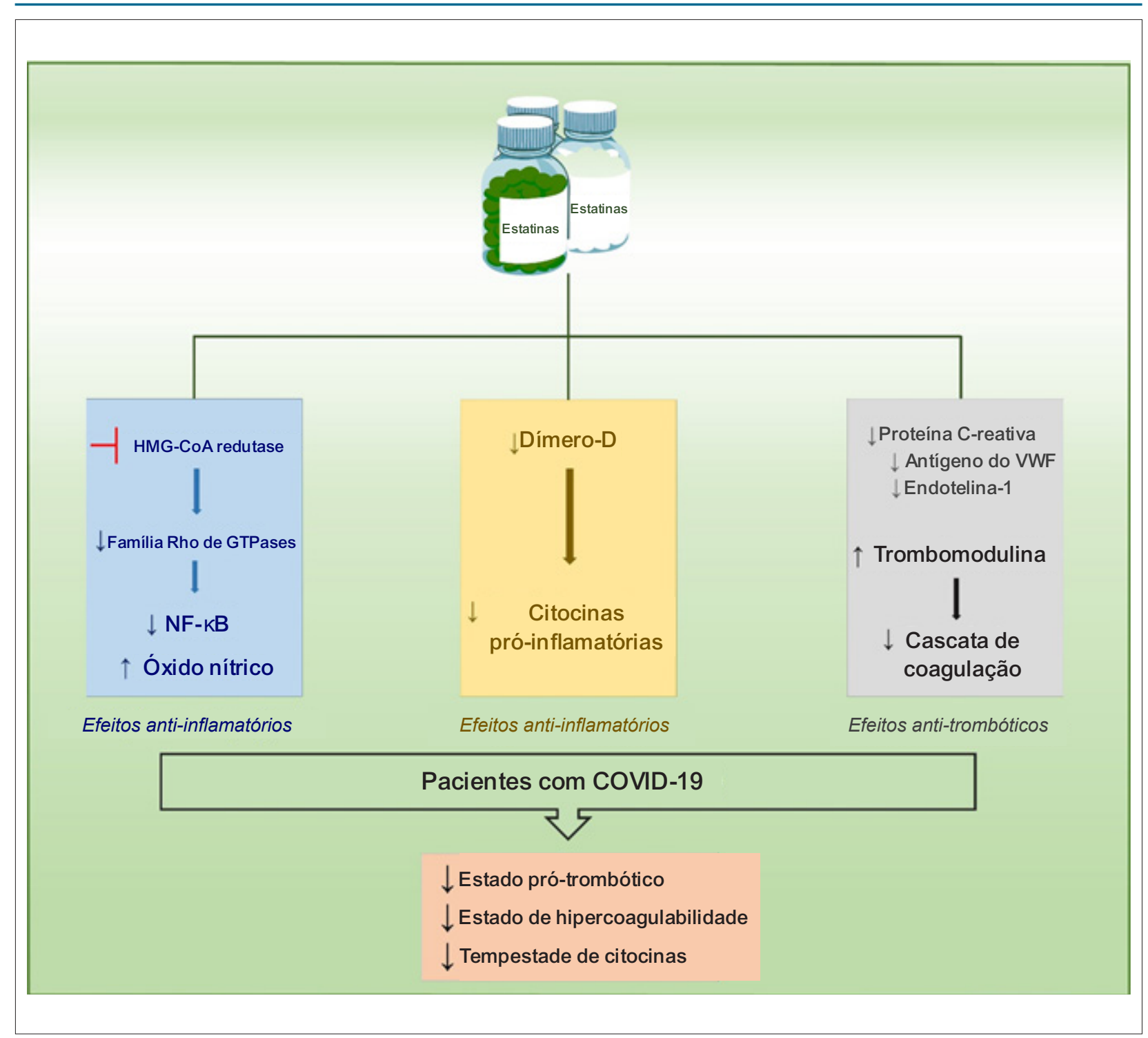

Figura 1-Alguns mecanismos propostos por meio dos quais as estatinas reduzem o estado pró-inflamatório e pro-trombótico em pacientes com COVID-19.8-11,24,25 HMG-CoA redutase: 3-hidroxi-3-methyl-glutaril-CoA redutase; NF-KB: fator nuclear kappa B; VWF: fator de von Willebrand.

Em suma, o uso de estatinas é baseado em uma literatura sólida e robusta, e a sua suspensão, exceto por indicação médica, pode levar a eventos agudos, aumentando ainda mais os riscos dos pacientes infectados por COVID-19, especialmente aqueles em prevenção secundária e aqueles que tiveram um evento coronário agudo recente. Os médicos e os pacientes devem manter esse conhecimento em mente.

\section{Quando Devemos Considerar a Suspensão das Estatinas em Pacientes com COVID-19?}

De acordo com as diretrizes da Sociedade Europeia de Cardiologia, em casos raros em que os pacientes com COVID-19 desenvolvem rabdomiólise grave ou aumento das enzimas hepáticas, a suspensão temporária da terapia com estatinas é prudente. ${ }^{31,32}$ Além disso, se o paciente estiver em risco iminente de vida, a suspensão deve ser realizada, pelo menos até a recuperação da infecção. ${ }^{33}$

\section{Conclusões}

O uso de estatinas é apoiado por uma literatura sólida, com inquestionáveis benefícios cardiovasculares. Apesar das evidências de que concentrações mais baixas de colesterol estão associadas a um curso mais grave de COVID-19, não há, entretanto, evidências de que as estatinas possam piorar o prognóstico. Ao contrário, essas drogas podem reduzir os mecanismos pró-inflamatórios e pró-trombóticos que caracterizam os casos mais graves de COVID-19. Atualmente, não existem evidências apoiando a descontinuação das estatinas em pacientes com COVID-19, exceto quando ocorrem elevações importantes das enzimas hepáticas, 
rabdomiólise ou risco de vida atribuído ao medicamento. Por outro lado, não há indicação para o uso desses medicamentos especificamente para prevenir complicações da infecção pelo SARS-CoV-2.

\section{Contribuição dos Autores}

Concepção e desenho da pesquisa, Obtenção de dados, Análise e interpretação dos dados e Redação do manuscrito: Ferrari F, Santos RD; Revisão crítica do manuscrito quanto ao conteúdo intelectual importante: Santos RD.

\section{Potencial Conflito de Interesses}

RDS recebeu honorários relacionados a atividades de consultoria, pesquisa e/ou palestras de: Aché, Amgen,
AstraZeneca, Esperion, Kowa, Novo Nordisk, Merck, MSD, Pfizer, PTC e Sanofi/Regeneron. FF declara não haver conflitos de interesse.

\section{Fontes de Financiamento}

Filipe Ferrari recebe suporte financeiro pela Coordenação de Aperfeiçoamento de Pessoal de Nível Superior - Brasil (CAPES) - Código 001. Raul D. Santos é bolsista do Conselho Nacional de Pesquisa e Desenvolvimento Tecnológico (CNPq), Brasil, no 303734/2018-3.

\section{Vinculação Acadêmica}

Não há vinculação deste estudo a programas de pósgraduação.

\section{Referências}

1. Wei X, Zeng W, Su J, Wan H, Yu X, Cao X, et al. Hypolipidemia is associated with the severity of COVID-19. J Clin Lipidol. 2020;14(3):297-304.

2. Fan J, Wang H, Ye G, Cao X, Xu X, Tan W, et al. Letter to the Editor: Lowdensity lipoprotein is a potential predictor of poor prognosis in patients with coronavirus disease 2019. Metabolism. 2020 Jun;107:154243.

3. South AM, Diz DI, Chappell MC. COVID-19, ACE2, and the cardiovascular consequences. Am J Physiol Heart Circ Physiol. 2020;318(5):H1084-90.

4. Alvarez C, Ramos A. Lipids, lipoproteins, and apoproteins in serum during infection. Clin Chem. 1986;32(1 Pt 1):142-5.

5. Sammalkorpi K, Valtonen V, Kerttula Y, Nikkila E, Taskinen MR. Changes in serum lipoprotein pattern induced by acute infections. Metabolism. 1988;37(9):859-65

6. Han R. Plasma lipoproteins are important components of the immune system. Microbiol Immunol. 2010;54(4):246-53

7. Libby P, Loscalzo J, Ridker PM, Farkouh ME, Hsue PY, Fuster V, et al. Inflammation, immunity, and infection in atherothrombosis: JACC Review Topic of the Week. J Am Coll Cardiol. 2018;72(17):2071-81.

8. Davignon J. Beneficial cardiovascular pleiotropic effects of statins. Circulation. 2004;109(23 Suppl 1):III39-43.

9. Genser B, Grammer TB, Stojakovic T, Siekmeier R, März W. Effect of HMC CoA reductase inhibitors on low-density lipoprotein cholesterol and C-reactive protein: systematic review and meta-analysis. Int J Clin Pharmacol Ther. 2008;46(10):497-510.

10. Sahebkar A, Serban C, Ursoniu S, Mikhailidis DP, Undas A, Lip GYH, et al. The impact of statin therapy on plasma levels of von Willebrand factor antigen. Systematic review and meta-analysis of randomised placebocontrolled trials. Thromb Haemost. 2016;115(3):520-32

11. Sahebkar A, Kotani K, Serban C, Ursoniu S, Mikhailidis D, Jones SR, et al Statin therapy reduce plasma endothelin-1 concentrations: a meta-analysis of 15 randomized controlled trials. Atherosclerosis. 2015;241(2):433-42.

12. Vandermeer ML, Thomas AR, Kamimoto L, Reingold A, Gershman K, Meek J, et al. Association between use of statins and mortality among patients hospitalized with laboratory-confirmed influenza virus infections: a multistate study. J Infect Dis. 2012;205(1):13-9.

13. Henry C, Zaizafoun M, Stock E, GhamandeS, Arroliga AC, White HD. Impact of angiotensin-converting enzyme inhibitors and statins on viral pneumonia. Proc (Bayl Univ Med Cent). 2018;31(4):419-23.

14. De Spiegeleer A, Bronselaer A, Teo JT, Byttebier G, Tré GD, Belmans $L$, et al. The Effects of ARBs, ACEis, and statins on clinical outcomes of COVID-19 infection among nursing home residents. J Am Med Dir Assoc. 2020;21(7):909-14.e2.
15. Zhang XJ, Qin JJ, Cheng X, Shen L, Zhao YC, Yuan Y, et al. In-hospital use of statins is associated with a reduced risk of mortality among individuals with COVID-19. Cell Metab. 2020;32(2):176-87.

16. Rodriguez-Nava G, Trelles-Garcia DP, Yanez-Bello MA, Chung CW, TrellesGarcia VP, Friedman HJ. Atorvastatin associated with decreased hazard for death in COVID-19 patients admitted to an ICU: a retrospective cohort study. Crit Care. 2020;24(1):429.

17. Daniels LB, Sitapati AM, Zhang J, Zou J, Bui QM, Ren J, et al. Relation of statin use prior to admission to severity and recovery among COVID-19 inpatients. Am J Cardiol. 2020 Dec 1;136:149-55.

18. Song SL, Hays SB, Panton CE, Mylona EK, Kalligeros M, Shehadeh F, et al Statin use is associated with decreased risk of invasive mechanical ventilation in COVID-19 patients: a preliminary study. pathogens. 2020;9(9):759.

19. Ridker PM, Danielson E, Fonseca FAH, Genest J, Gotto Jr AM, Kastelein JJP, et al. Rosuvastatin to prevent vascular events in men and women with elevated C-reactive protein. N Engl J Med. 2008;359(21):2195-207.

20. Novack V, MacFadyen J, Malhotra A, Almog Y, Glynn RJ, Ridker PM. The effect of rosuvastatin on incident pneumonia: results from the JUPITER trial. CMAJ. 2012;184(7):E367-72.

21. Helms J, Tacquard C, Severac F, Lorant IL, Ohana M, Delabranche $X$, et al. High risk of thrombosis in patients with severe SARS-CoV-2 infection: a multicenter prospective cohort study. Intensive Care Med. 2020;46(6):1089-98

22. Glynn RJ, Danielson E, Fonseca FA, Genest I, Gotto Jr AM, Kastelein JJP, et al. A randomized trial of rosuvastatin in the prevention of venous thromboembolism: the JUPITER trial. N Engl J Med. 2009;360(18):1851-61.

23. Kunutsor SK, Seidu S, Khunti K. Statins and primary prevention of venous thromboembolism: a systematic review and meta-analysis. Lancet Haematol. 2017;4(2):e83-93.

24. Adams NB, Lutsey PL, Folsom AR, Herrington DH, Sibley CT, Zakai NA, et al Statin therapy and levels of hemostatic factors in a healthy population: the Multi-Ethnic Study of Atherosclerosis. J Thromb Haemost. 2013;11(6):107884.

25. Rodriguez AL, Wojcik BM, Wrobleski SK, Myers Jr DD, Wakefield TW, Diaz JA. Statins, inflammation and deep vein thrombosis: a systematic review. J Thromb Thrombolysis. 2012;33(4):371-82

26. Bertoletti L, Couturaud F, Montani D, Parent F, Sanchez O. Venous thromboembolism and COVID-19. Respir Med Res. 2020 Nov; 78:100759.

27. Cholesterol Treatment Trialists' (CTT) Collaboration, Baigent C, Blackwell L, Emberson J, Holland LE, Reith C, et al. Efficacy and safety of more intensive lowering of LDL cholesterol: a meta-analysis of data from 170,000 participants in 26 randomised trials. Lancet. 2010;376(9753):1670-81. 


\section{Carta Científica}

28. Sposito AC, Carvalho LS, Cintra RM, Araújo ALR, Ono AH, Andrade JM, et al. Rebound inflammatory response during the acute phase of myocardial infarction after simvastatin withdrawal. Atherosclerosis. 2009;207(1):191-4.

29. PRISM Investigators. A comparison of aspirin plus tirofiban with aspirin plus heparin for unstable angina: the Platelet Receptor Inhibition in Ischemic Syndrome Management (PRISM) Study Investigators. N Engl J Med. 1998;338(21):1498-505

30. Heeschen C, Hamm CW, Laufs U, Snapinn S, Böhm M, White HD, et al. Withdrawal of statins increases event rates in patients with acute coronary syndromes. Circulation. 2002;105(12):1446-52.
31. European Society of Cardiology. ESC Guidance for the Diagnosis and Management of CV Disease during the COVID-19 Pandemic; 2020. [citado 31 jul. 2020]. Disponível em: <https://www.escardio.org/Education/ COVID-19-and-Cardiology/ESC-COVID-19-Guidance $>$.

32. Subir R, Jagat JM, Kalyan KG. Pros and cons for use of statins in people with coronavirus disease-19 (COVID-19). Diabetes Metab Syndr. 2020;14(5):1225-9.

33. Greenhalgh T, Koh GCH, Car J. Covid-19: a remote assessment in primary care. BMJ. 2020 Mar 25;368:m1182.

Este é um artigo de acesso aberto distribuído sob os termos da licença de atribuição pelo Creative Commons 